EDITORIAL

\section{What causes lacunar stroke?}

\section{J M Wardlaw}

A quarter of all ischaemic strokes (a fifth of all strokes) are lacunar type. ${ }^{1}$ Lacunar infarcts are small infarcts (2-20 $\mathrm{mm}$ in diameter) in the deep cerebral white matter, basal ganglia, or pons, presumed to result from the occlusion of a single small perforating artery supplying the subcortical areas of the brain. ${ }^{2}$ Although a recognised stroke subtype for over 50 years, the cause of lacunar ischaemic stroke, ${ }^{2}$ and whether it is different to cortical ischaemic stroke, remains under debate. ${ }^{3}$ Furthermore, lacunar stroke is not benign; $30 \%$ of patients are left dependent, ${ }^{5}$ and scant long term data suggest that up to $25 \%$ of patients have a second stroke within 5 years. ${ }^{6}$ Therefore, the prevention and treatment of this common stroke subtype may be less than ideal.

\section{PROBLEMS IN THE STUDY OF LACUNAR STROKE}

Several factors have hampered the study of lacunar stroke. Firstly, few patients die from lacunar stroke; if they do, death may occur long after the stroke, making autopsy material scant and difficult to interpret, and the small cerebral vessels require meticulous dissection. Studies of risk factors and causation have predominantly used a clinical diagnosis of stroke type, probably resulting in some misclassification. Although lacunar infarcts are associated with specific neurological syndromes, and most patients with a clinical lacunar syndrome have a small deep subcortical infarct on brain imaging (if visible), $10-20 \%$ actually have a recent small cortical infarct in a location that explains their stroke presentation. ${ }^{7}$ Similarly, $10-20 \%$ of patients with a clinical mild cortical stroke actually have a recent relevant lacunar infarct on imaging. ${ }^{7}$ Epidemiologically, these patients behave more like the lesion type on imaging than the clinical syndrome of the lesion they actually have. ${ }^{7}$ Many studies have used an inappropriate control group; age matched normal controls can only indicate whether or not a particular risk factor is associated with stroke, whereas identification of associations specific to lacunar stroke requires a control group with a different type of ischaemic stroke. Finally, some classifications, such as the Trial of Org 10172 in Acute Stroke Treatment (TOAST) method $^{8}$ actually use risk factors (such as embolic sources or hypertension) to determine the stroke type, thus potentially biasing studies of differences in risk factors. Hence, inadvertent misdiagnosis of lacunar as cortical stroke, and vice versa, the paucity of pathological material, and bias in some clinical classification systems may have confounded previous pathology, prognosis, and risk factor studies.

\section{IS THE CAUSE OF LACUNAR DIFFERENT FROM CORTICAL ISCHAEMIC STROKE?}

The lacunar hypothesis supports the concept that lacunar ischaemic stroke is due to an intrinsic cerebral small arteriolar abnormality, ${ }^{2}$ in contrast to cortical ischaemic stroke, which is commonly due to embolism from the heart or large arteries. Although some studies suggest that many lacunar strokes are caused by emboli, and while it is perfectly possible for a small embolus to enter and occlude a lenticulostriate artery, ${ }^{9}$ a systematic review of risk factors including only studies using subtype definitions for ischaemic stroke free of risk factors found that atrial fibrillation and carotid stenosis were associated more with non-lacunar than lacunar infarction (relative risk (RR) of lacunar versus non-lacunar infarction: atrial fibrillation $0.51,95 \%$ confidence interval (CI) 0.42 to 0.62 ; ipsilateral carotid stenosis $0.35,95 \%$ CI 0.28 to $0.44) .{ }^{10}$ Indeed, common causes of large artery (cortical) infarction, such as emboli from the large arteries or heart, ${ }^{11-13}$ or intracranial large artery atheromatous stenoses, ${ }^{12}$ appear unlikely to cause more than $10-15 \%$ of lacunar strokes. ${ }^{14-17}$ Perhaps lacunar infarcts due to emboli or middle cerebral artery stenoses are recognisable by being larger than non-embolic/stenotic lacunes, ${ }^{12}{ }^{18}$ possibly because the embolus/stenosis occluded the origin of several lenticulostriate arterioles simultaneously. There is a suggestion that lacunar stroke secondary to middle cerebral artery stenosis may be more common in south Asian populations than in western white populations, but this remains to be clarified. Only $6 \%$ of all particles (even the smallest) injected into the carotid arteries in an experimental model ended up in the lenticulostriate arteries, the rest going to the cortical arteries or their cortical branches. ${ }^{9}$ Studies that suggested stronger associations between lacunar stroke and emboli cited carotid stenoses as mild as $25 \%{ }^{16}$ or cardiac abnormalities not clearly associated with embolism (for example, left ventricular hypertrophy), ${ }^{13}$ or they had no, or an inappropriate control group. It would certainly be useful to be able to infer the likely underlying mechanism of lacunar ischaemic stroke (that is, to identify the $10-15 \%$ of embolic/stenotic versus other intrinsic small vessel strokes) from the appearance of the brain lesion, as that might help target effective secondary prevention regimens, but much more work is required to see whether different patterns actually exist, before determining how closely these relate to the underlying mechanism.

Hypertension and diabetes are said to be strongly associated with lacunar ischaemic stroke. However, in studies using risk factor free ischaemic stroke subtype definitions, there was only a marginal excess of hypertension with lacunar versus non-lacunar infarction (RR 1.11; 95\% CI 1.04 to 1.19 ) and no difference for diabetes (RR 0.95; 95\% CI 0.83 to 1.09$).{ }^{10}$ Nor was there clear evidence of any association between smoking, prior transient ischaemic attack, excess alcohol consumption, or raised cholesterol in lacunar compared with non-lacunar infarction. ${ }^{10}$

\section{IS THERE OTHER EVIDENCE OF A DIFFERENT MECHANISM IN LACUNAR ISCHAEMIC STROKE?}

After lacunar ischaemic stroke, a recurrence is more likely to be lacunar then cortical; $47 \%$ of recurrences after a lacunar stroke were lacunar compared with $15 \%$ of recurrences after a nonlacunar stroke). ${ }^{19}$ If lacunar and cortical ischaemic strokes were due to the same mechanisms, then recurrent strokes would be equally likely to be cortical after a lacunar stroke as lacunar, which appears not to be the case.

Lacunar ischaemic stroke also appears to be more closely associated with white matter lesions (WMLs) than does cortical ischaemic stroke. WMLs are abnormal areas of hypodensity (on computed tomography scans) or hyperintensity (on T2 weighted magnetic resonance imaging (MRI)) in the deep hemispheric and periventricular white matter and brain stem. ${ }^{20}$ They are in turn associated with cognitive decline, ${ }^{21}$ and 
increased risk of future stroke, particularly lacunar type. ${ }^{22}$ WMLs also progress rapidly after lacunar stroke. ${ }^{23}$ After lacunar stroke, 15-20\% develop dementia (more than might reasonably be attributed to the amount of brain damage caused by a lacunar infarct). ${ }^{621} 24$ After lacunar ischaemic stroke, new "silent lacunar infarcts" occur on follow up imaging. ${ }^{25}$ Asymptomatic small deep white matter infarcts, in addition to the symptomatic lesion, have been seen on MR diffusion imaging at presentation with lacunar ischaemic stroke. ${ }^{26}$ The imaging appearance of these asymptomatic lacunar infarcts suggests that they are slightly older than the presenting lesion. Cerebral microhaemorrhages, which are tiny, apparently asymptomatic bleeds (or "leaks") in the brain are also associated with lacunar stroke and WMLs. ${ }^{27}$ These observations suggest that most lacunar strokes are the clinically focal manifestation of what is actually a diffuse abnormality of the small cerebral arterioles, which, if extensive enough, can also manifest clinically as cognitive decline and dementia. ${ }^{28}$

\section{WHAT IS THE CEREBRAL SMALL VESSEL ABNORMALITY?}

Detailed pathology studies in the 1950s identified abnormal small deep perforating (lenticulostriate) arteries resulting in lacunar infarcts, ${ }^{29}$ termed segmental arterial wall disorganisation (since called lipohyalinosis or fibrinoid necrosis). In lipohyalinosis, the vessel wall appears thickened, with focal dilation, and eventually leads to disintegration of the wall with an infarct around it. This arteriolar abnormality remains the most commonly described defect to date. ${ }^{30} 31$ However, there is debate about the pathology and its relationship to symptoms; many studies did not ascertain that the lesion seen at autopsy was symptomatic, some studied multiple small "holes" in the brain regardless of symptoms, and many lesions came from few patients. ${ }^{32}$ Lipohyalinosis is found in areas of the brain corresponding with WMLs on imaging. ${ }^{33}$

The nature of the intrinsic arteriolar abnormality remains unresolved. It may be microatheroma, poor cerebral blood flow, or vasospasm. It is difficult to see why atheroma would affect the small arterioles when there is a lack of association with the better understood large artery atheroma in lacunar infarction, ${ }^{10}{ }^{14}$ Vasospasm, inducible in animal models with extreme hypertension, causes fibrinoid necrosis, but the association of lacunar infarction with any excess of hypertension (more than other types of ischaemic stroke) in patients is weak (RR 1.11; 95\% CI 1.04 to 1.19 )..$^{10}$ Thickened vessels may narrow or occlude the lumen, leading to ischaemia, but relatively few truly occluded vessels have been seen pathologically. ${ }^{29}{ }^{30}$ Thickened vessel walls may be stiff and impair autoregulation, and indeed patients with WMLs may have impaired autoregulation. ${ }^{34}$ Some studies found reduced cerebral blood flow $(\mathrm{CBF})$ in patients with WMLs, ${ }^{35}$ but others did not. ${ }^{36} \mathrm{CBF}$ is difficult to quantify: "reduced" CBF may be artefactual, ${ }^{37}$ or simply the consequence of a reduction in normal tissue to supply. None of this explains what starts the vessel abnormality, only the features or behaviour of the vessels and the possible mechanisms for damaging the brain, once the abnormality is established.

\section{IF NOT OCCLUSION AND ISCHAEMIA, THEN WHAT?}

The underlying abnormal lenticulostriate artery can be seen in about $10 \%$ of lacunar infarcts on detailed MRI scans. ${ }^{38}$ Although this appearance could be an intra-luminal thrombus (a small arteriolar "hyperdense middle cerebral artery sign" equivalent), other features suggest that blood products had passed into the vessel wall and perivascular space. The infarcts appear to be around, rather than at the end of the abnormal segment. Lesions that resemble an "incomplete" lacunar infarct (perivascular oedema related lesions) at autopsy $^{39}$ suggest that the "infarct" was actually due to oedema fluid leaking from the arteriole and damaging adjacent tissue.

Increased "leakage" of intravenously injected MR contrast agent across the blood-brain barrier have been found on detailed MR imaging in patients with WMLs. ${ }^{40}$ There are numerous other examples in a rather scattered literature pointing to "leaky" small arterioles predisposing to WMLs and lacunar ischaemic stroke, of which the following are but a few. Extravasated plasma proteins have been found at autopsy in WMLs in patients with ischaemic cerebrovascular disease in life. ${ }^{41}$ Discrete areas of extravasated plasma proteins have been seen around small cerebral arterioles in hypertensive primates. ${ }^{42}$

These combined observations suggest that the initiating step for most lacunar ischaemic stroke and WMLs may be failure of the arteriolar endothelium (that is, the blood-brain barrier) allowing extravasation of blood components into the vessel wall, and consequently vessel wall, perivascular neuronal, and glial cell damage. ${ }^{28} 394142$ This would explain the features described histologically, ${ }^{293132}$ and the association with microhaemorrhages (small blood leaks). ${ }^{27}$

Is there other evidence of endothelial failure? Patients with isolated lacunar infarction, or lacunar infarction plus WMLs, have elevated systemic plasma markers of endothelial activation (plasma intercellular adhesion molecule 1 , thrombomodulin, and tissue factor pathway inhibitor) compared with age matched normal controls. ${ }^{43}$ However, in the absence of non-lacunar controls, it is unclear if these patterns are specific to lacunar stroke. Several studies have identified retinal microvascular abnormalities that were associated with increased risk of stroke, cognitive impairment, and white matter lesions. Those most closely related to these cerebral abnormalities (microaneurysms, retinal haemorrhages, and soft exudates) were most commonly seen when there was breakdown of the blood-retinal barrier. ${ }^{45}$ Unfortunately most of these studies did not examine stroke subtypes, so more work is required.

\section{SUMMARY}

The mechanism underlying, and the long term consequences of, lacunar ischaemic stroke, the cause of a quarter of all ischaemic strokes, are poorly understood, but are not benign. Evidence supports the hypothesis that, in most lacunar strokes, the vascular abnormality is pathologically diffuse, even if the clinical manifestations are focal, and result from small vessel endothelial damage, subtle increase in blood-brain barrier permeability, and leakage of substances toxic to the brain into the perivascular tissue. As originally proposed in the lacunar hypothesis, only a small proportion of lacunar stroke appears to result from artery to artery or cardiogenic emboli or intracranial large artery stenoses. These latter embolic/ stenotic lesions may be recognisable by their size (being larger).

It might be questioned how this presumably gradual process could produce a sudden lacunar infarct. In fact, lacunar stroke symptoms (more than other stroke subtypes) not uncommonly progress after onset. ${ }^{44}$ Perhaps the interstitial fluid composition eventually reaches a critically abnormal point where the axons cease to transmit signals, or the vessel wall thickening narrows the lumen and reduces blood flow, or fails to allow nutrients out and waste products of metabolism back into the circulation. This diffuse endothelial failure might also account for additional asymptomatic lacunar infarcts observed at presentation with, ${ }^{26}$ or during follow up after, ${ }^{25}$ a symptomatic lacunar infarct with no underlying embolic source. 
These asymptomatic lesions, gradually multiplying, could be the source of the WMLs. Neurones can switch off suddenly, even when the underlying process is gradual; for example, neurones switch off electrical activity (manifest as a stroke) as cerebral blood flow falls below about $25 \mathrm{ml} / 100 \mathrm{~g}$ brain/min, thus the fall in blood flow may have been gradual but the symptoms are sudden.

An important target for new therapeutic approaches to prevent or treat a common form of ischaemic stroke and cognitive decline may have been overlooked. It is important to understand this process better, not simply in order to reduce the burden of lacunar stroke, but because the same mechanism may also underlie WMLs and consequent cognitive decline to dementia. Lacunar ischaemic stroke has for too long been simply lumped together with other stroke subtypes; while it is likely that many older stroke patients will share common vascular risk factors, this tendency has hindered understanding of what appears to be an importantly different stroke subtype that may require different acute treatment and prevention. For example, the association with microhaemorrhages, a possible risk factor for intracerebral haemorrhage, is worrying, and may increase the risk of haemorrhage complicating anti-thrombotic drug treatment compared with their use in cortical ischaemic stroke. Further studies, using detailed, accurate, and unbiased patient classification, are needed to examine risk factors for lacunar stroke including blood-brain barrier function (now possible with detailed MRI), ${ }^{40}$ how to identify those lacunar strokes that are due to embolism, and clarify long term outcomes. Study of small arteriolar abnormalities in other vascular beds (for example the retina, where the arterioles can be directly observed) and systemic endothelial abnormalities will help both in understanding the mechanisms of lacunar ischaemic stroke and in identifying diagnostic and prognostic markers.

\section{ACKNOWLEDGEMENTS}

I would like to acknowledge the many people who have discussed with me the possible mechanisms of lacunar ischaemic stroke or authored some of the supporting papers, in particular Professor C Warlow, Professor M Dennis, Dr C Sudlow, Dr A Lammie, Dr J Bamford, Professor P Sandercock, Dr P Armitage, and Dr G Mead.

J Neurol Neurosurg Psychiatry 2005;76:617-619.

doi: 10.1136/jnnp.2004.039982

Correspondence to: Dr J M Wardlaw, Division of Clinical Neurosciences, Western General Hospital, Crewe Rd, Edinburgh EH4 2XU UK; joanna.wardlaw@ed.ac.uk
Received 11 November 2004

In revised form 29 November 2004

Accepted 1 December 2004

Competing interests: none declared

\section{REFERENCES}

1 Sudlow CLM, Warlow CP. Comparable studies of the incidence of stroke and its pathological types. Results from an international collaboration. Stroke 1997;28:491-9.

2 Bamford JM, Warlow CP. Evolution and testing of the lacunar hypothesis. Stroke 1988; 19:1074.

3 Futrell N. Lacunar infarction. Embolism is the key. Stroke 2004;35:1778-9.

4 Norrving B. Lacunar infarction. Embolism is the key: Against. Stroke 2004;35:1779-80.

5 Bamford J, Sandercock P, Dennis M, et al. Classification and natural history of clinically identifiable subtypes of cerebral infarction. Lancet 1991:337:1521-6.

6 Samuelsson M, Soderfeldt B, Olsson GB. Functional outcome in patients with lacunar infarction. Stroke 1996;27:842-6.

7 Mead GE, Lewis S, Wardlaw JM, et al. Should computed tomography appearance of lacunar stroke influence patient management? J Neurol Neurosurg Psychiatry 1999;67:682-4.

8 Adams HP Jr, Bendixen BH, Kappelle $\amalg$, et al. Classification of subtype of acute ischemic stroke. Definitions for use in a multicenter clinical trial. TOAST. Trial of Org 10172 in Acute Stroke Treatment. Stroke 1993;24:35-41

9 Macdonald RL, Kowalczuk A, Johns L. Emboli enter penetrating arteries of monkey brain in relation to their size. Stroke 1995;26:1247-51.

10 Jackson C, Sudlow C. Are lacunar strokes really different? A systematic review of differences in risk factor profiles between lacunar and nonlacunar infarcts. Stroke 2004. In press.

11 Adachi T, Kobayashi S, Yamaguchi S, et al. MRI findings of small subcortical "lacunar-like" infarction resulting from large vessel disease. J Neurol 2002;247:280-5.

12 Baumgartner RW, Sidler C, Mosso M, et al. Ischemic lacunar stroke in patients with and without potential mechanism other than smallartery disease. Stroke 2003;34:653-9.

13 Kazui S, Levi CR, Jones EF, et al. Lacunar stroke: Transoesophageal echocardiographic factors influencing long-term prognosis. Cerebrovasc Dis 2001:12:325-30.

14 Mead GE, Lewis SC, Wardlaw JM, et al. Severe ipsilateral carotid stenosis and middle cerebral artery disease in lacunar ischaemic stroke: innocent bystanders? J Neurol 2002;249:266-71

15 Ay H, Oliveira-Filho J, Buonanno FS, et al Diffusion-weighted imaging identifies a subset of lacunar infarction associated with embolic source. Stroke 1999:30:2644-50.

16 Uehara T, Tabuchi M, Mori E. Occlusive lesions of carotid and intracranial arteries in patients with symptomatic lacunar infarction. Evaluation by MR angiography. Clin Neurol 1997;37:796-801.

17 Tejada J, Diez-Tejedor E, HernandezEchebarria L, et al. Does a relationship exist between carotid stenosis and lacunar infarction? Stroke 2003;34:1404-11.

18 de Jong G, Kessels F, Lodder J. Two types of lacunar infarcts: further arguments from a study on prognosis. Stroke 2002;33:2072-6.

19 Jackson C, Sudlow C. Risks of death and recurrent vascular events after lacunar and nonlacunar ischaemic stroke (LACl and non-LACI) - a systematic review of follow-up studies. Cerebrovasc Dis 2004;17(suppl 5):52.

20 Inzitari D. Leukoaraiosis. An independent risk factor for stroke? Stroke 2003;34:2071.

21 Schmidt R, Enzinger C, Ropele S, et al. Progression of cerebral white matter lesions: 6 year results of the Austrian Stroke Prevention Study. Lancet 2003;361:2046-8.

22 Kuller LH, Longstreth WT Jr, Arnold AM, et al. White matter hyperintensity on cranial magnetic resonance imaging. A predictor of stroke. Stroke 2004;35:1821-3
23 Samuelsson $M$, Lindell D, Olsson G-B. Lacunar infarcts: A 1-year clinical and MRI follow-up study. Cerebrovasc Dis 1994:4:265-72.

24 Voisin T, Rous de Feneyrols AR, Pavy Le Traon A, et al. Cognitive impairment after first lacunar stroke: clinical features and risk factors. Cerebrovasc Dis 2002;13(suppl 3):297.

25 O'Sullivan M, Rich PM, Barrick TR, et al. Frequency of subclinical lacunar infarcts in ischemic leukoaraiosis and cerebral autosomal dominant arteriopathy with subcortical infarcts and leukoencephalopathy. Am J Neuroradiol 2003;24:1348-54.

26 Chowdhury D, Wardlaw JM, Dennis MS. Are multiple lacunar infarctions caused by embolic mechanisms? J Neurol Neurosurg Psychiatry 2004;75. In press

27 Kato H, Izumiyama M, Izumiyama K, et al. Silent cerebral microbleeds on T2*-weighted MRI: correlation with stroke subtype, stroke recurrence, and leukoaraiosis. Stroke 2002;33:1536-40.

28 Wardlaw JM, Sandercock PA, Dennis MS, et al. Is breakdown of the blood-brain barrier responsible for lacunar stroke, leukoaraiosis, and dementia? Stroke 2003;34:806-12.

29 Fisher CM. Lacunes: Small, deep cerebral infarcts. Neurology 1965;15:774-84.

30 Arboix A, Marti-Vilalta JL. Estudio de los infartos lacunares a partir del analisis de las principales series anatomopatologicas de la literatura. Rev Neurol 1998;26:365-7.

31 Lammie GA. Pathology of small vessel stroke. $\mathrm{Br}$ Med Bull 2000;56:296-306.

32 Fisher $\mathrm{CM}$. The arterial lesions underlying lacunes. Acta Neuropath 1969;12:1-15.

33 Pantoni L. Pathophysiology of age-related cerebral white matter changes. Cerebrovasc Dis 2002;13(suppl 2):7-10

34 Bakker SLM, de Leeuw F-E, de Groot JC, et al. Cerebral vasomotor reactivity and cerebral white matter lesions in the elderly. Neurology 1999;52:578-83.

35 O'Sullivan M, Lythgoe DJ, Pereira AC, et al. Patterns of cerebral blood flow reduction in patients with ischemic leukoaraiosis. Neurology 2002;59:321-6

36 Yao H, Yuzuriha T, Fukuda K, et al. Cerebral blood flow in nondemented elderly subjects with extensive deep white matter lesions on magnetic resonance imaging. J Stroke Cerebrovasc Dis 2000;9:172-5

37 Blamire A, Styles P. Does contrast based perfusion imaging give the right answer in pathological tissue? A Monte Carlo simulation study. Proc Intl Soc Mag Reson Med 2001;9:1586

38 Wardlaw JM, Dennis MS, Warlow CP, et al. Imaging appearance of the symptomatic perforating artery in patients with lacuna infarction: occlusion or other vascular pathology? Ann Neurol 2001;50:208-15.

39 Lammie GA, Brannan F, Wardlaw JM. Incomplete lacunar infarction (type $1 \mathrm{~b}$ lacunes). Acta Neuropath 1998:96:163-71.

40 Starr JM, Wardlaw J, Ferguson K, et al. Increased blood-brain barrier permeability in type II diabetes demonstrated by gadolinium magnetic resonance imaging. JNNP 2003:74:70-6.

41 Tomimoto H, Akiguchi I, Suenaga T, et al. Alterations of the blood-brain barrier and glial cells in white matter lesions in cerebrovascular and Alzheimer's disease patients. Stroke 1996;27:2069-74.

42 Kemper TL, Blatt GJ, Killiany RJ, et al. Neuropathology of progressive cognitive decline in chronically hypertensive rhesus monkeys. Acta Neuropathol (Berl) 2001; 101:145-53.

43 Hassan A, Hunt BJ, O'Sullivan M, et al. Markers of endothelial dysfunction in lacunar infarction and ischaemic leukoaraiosis. Brain 2003; 126:424-32

44 Nakamura K, Saku Y, Ibayashi S, et al. Progressive motor deficits in lacunar infarction. Neurology 1999:52:29-33.

45 Wong TY. Is retinal photography useful in the measurement of stroke risk? Lancet Neurol 2004;3:179-83. 


\section{Imaging Huntington's disease (HD) brains - imagine HD trials!}

\section{H P H Kremer}

\section{Tensor based morphometry}

T he true and ultimate aim of research in Huntington's disease (HD) is to arrest the progressive neurodegeneration and thus the debilitating clinical and functional deterioration-or so it seems to those involved in the clinical care of the unfortunate individuals affected by it. Two major clinical challenges confront us: to retard, or better, to abolish disease progression in symptomatic individuals; and to prevent phenoconversion in presymptomatic individuals.

Huntington's disease is considered to be rare, but extrapolation of its estimated prevalence-in western countries 5 to 10 per 100000 -yields for the current 25 countries of the European Union (with 455 million people in 2004) a total number of between 22500 and 45000 clinically affected patients in various stages of the disease. The number of presymptomatic mutation carriers is much higher. Recent advances in our understanding of the molecular pathobiology have raised hopes that rational treatments may be near. Huntington's disease is caused by an expanded CAG triplet repeat in exon 1 of the huntingtin gene, and this mutation is expressed and translated into an expanded polyglutamine sequence in about half of all huntingtin protein molecules that are produced in the body cells. The physiological functions of the protein are still unknown. But many details of huntingtin interactions, cleavage, conformational changes, aggregation, and proteasomal breakdown have been clarified in the past decade. ${ }^{1}$ Based upon these mechanistic models, various potential drugs have been proposed. In fact, efficacy of such proposals has been demonstrated in simple cellular or more complex animal models, such as transgenic flies or mice. ${ }^{2}$ Another approach has been the large scale screening of promising compounds in simple systems ${ }^{3}$; and even older hypotheses regarding the causes of neurodegeneration, such as excitotoxicity, have yielded proposals for neuroprotective treatments. In fact, most of the past and ongoing neuroprotection trials in HD have studied compounds derived from this hypothetic excitotoxicity.

But these trials have faced one major problem. All of them have used serial clinical or functional assessments of symptomatic patients as the primary outcome measure. Despite their demonstrated robustness, linearity, and relevance, the intrinsic variability of clinical outcome measures in treated cohorts has been large. As a result, phase II and III neuroprotection trials have typically included relatively large numbers of symptomatic patients who were followed for several years. Thus a North American trial which studied the effects of remacemide and coenzyme Q (CoQ) against placebo in a $2 \times 2$ factorial design enrolled 347 patients and followed them for 30 months. The study failed to show any benefits of the treatment, although a trend towards an effect of CoQ has been suggested. ${ }^{4}$ Based upon this study, calculations predicted that a sufficiently powered CoQ trial would require more than 1000 patients to be followed for at least two years. The European EHDI trial that studies the effects of riluzole on disease progression in a 1:2 randomisation design enrolled 560 patients who were followed for 37 months. Results of this trial are pending. Previous interventions which studied baclofen, idebenone, vitamin $\mathrm{E}$, and lamotrigine included 100 patients or fewer, but all of them turned out to be clearly underpowered. The lesson: Huntington trials need large numbers of participating patients, to be followed for years.

If we were to conduct 10 such European protection trials simultaneously, about a quarter of all the patients in the $25 \mathrm{EU}$ countries would have to be enrolled and followed for many years. If we were to conduct an intervention in presymptomatic patients, the difficulties would be compounded because of the very long follow up times required to demonstrate phenoconversion. The logistics issue then becomes: how can we persuade sufficient participants and generate resources for such trials? Shorter trials that require fewer patients would clearly be preferable. The statistical answer would be: let us reduce end point variability.

Enter the paper by Kipps et al ${ }^{5}$ (this issue, $p p$ 650-5), about imaging the structural disease progression in preclinical disease. Using a novel approach to statistical imaging, called tensor based morphometry, they were able to demonstrate over a period of two years a progressive regional grey matter loss in 17 presymptomatic Huntington mutation carriers compared with 13 mutation negative controls. In contrast, clinical measurements failed to pick up any deterioration. If this result can be confirmed by others, imaging would become a powerful tool in neuroprotection trials. Imagine an intervention trial in which 40 patients are randomised and followed for two years, with, as the major measurements, an MRI scan at baseline and at the end of the trial. Although such measurements would have to be considered surrogate end points, they would nevertheless assume a crucial role in the selection of compounds to undergo the final test of clinical efficacy: a randomised controlled clinical trial with 1000 patients enrolled and followed for 30 months, with clinical outcome as the primary end point. We could certainly start planning a "primary prevention" trial aimed at postponing (or abolishing?) the onset of the disease.

J Neurol Neurosurg Psychiatry 2005;76:620. doi: 10.1136/jnnp.2004.056267

Correspondence to: $\mathrm{Dr} \mathrm{H}$ P H Kremer, Department of Neurology 326, PO Box 9101 6500 HB Niimegen, Netherlands; h.kremer@ neuro.umcn.nl

Competing interests: none declared

\section{REFERENCES}

1 Bates G. Huntingtin aggregation and toxicity in Huntington's disease. Lancet 2003:361:1642-44.

2 Steffan JS, Bodai L, Pallos J, et al. Histone deacetylase inhibitors arrest polyglutaminedependent neurodegeneration in Drosophila. Nature 2001;413:739-43.

3 Heiser V, Engemann S, Brocker W, et al. Identification of benzothiazoles as potential polyglutamine aggregation inhibitors of Huntington's disease by using an automated filter retardation assay. Proc Natl Acad Sci USA 2002;99(suppl 4):16400-6.

4 Huntington Study Group. A randomized, placebo-controlled trial of coenzyme Q10 and remacemide in Huntington's disease. Neurology 2001;57:397-404.

5 Kipps CM, Duggins AJ, Mahant N, et al. Progression of structural neuropathology in preclinical Huntington's disease: a tensor based morphometry study. I Neurol Neurosurg Psychiatry 2005;76:650-5. 


\section{Weight bearing asymmetry in standing hemiparetic patients}

\section{Pérennou}

\section{MCA stroke may disrupt corticobulbar projection to brainstem output pathways involved in the vestibular control of balance}

$\mathrm{P}$ stural disorders are a primary disability after stroke. ${ }^{1}$ They lead to loss of autonomy and expose patients to a high risk of falling. We must bear in mind that following a total anterior circulation infarct, the median time to recover the ability to stand for $10 \mathrm{~s}$ is 44 days (25th-75th percentile: 38-57 days $)^{2}$. Retraining the patient to stand is therefore a primary goal in post stroke rehabilitation, especially following hemisphere strokes.

Three main patterns characterise the standing posture of hemiparetic patients $^{1}$ : i) an increase in centre of gravity displacement, which reflects postural instability and results from orthopaedic, sensorimotor, and cognitive impairments; ii) the presence of a small area of stability beyond which the centre of gravity cannot move without exposing the patient to a loss of balance (this results either from an inability to control a stressed equilibrium system or from impaired co-ordination between posture and movement); and iii) weight bearing asymmetry, with more weight on the non-paretic leg. Unstable standing posture, although a major target in stroke rehabilitation, is still poorly understood. Weakness certainly plays a role, as do cognitive disorders observed in patients with lesions of the right hemisphere. ${ }^{3}$ These cognitive disorders result in distortion of the coordinates used to distribute loading over the two legs while standing. Since some patients align their erect posture to a biological vertical contralesionally tilted, it has also been suggested that the shift in the center of gravity towards the ipsilesional leg might be a compensatory strategy to prevent contralesional falling. ${ }^{1}$

In a very elegant experimental study published in this issue $(p p$ 670-8), Marsden et al propose a new approach to the problem of postural instability in standing hemiparetic patients. Using the measurement of forces and movements elicited by galvanic and transcranial electrical stimulation, they have explored the possibility of asymmetric vestibulo-spinal excitability in chronic middle cerebral artery (MCA) stroke patients, in patients with isolated corticospinal tract lesions, and in normal subjects. Patients and subjects were required to stand barefoot on two separate force plates with equal weight on both legs and with eyes closed. One advantageous feature of galvanic vestibular stimulation is that it is possible to evoke a bilateral response by stimulating vestibular afferents on one side only, ${ }^{4}{ }^{5}$ which means any asymmetries that may exist in the response pattern can be identified. It also offers an opportunity to decide whether asymmetry arises from an abnormality in the processing of sensory information (altered response in both legs for a lateralised deficit of sensory information) or an abnormality in the motor control of one side of the body (altered response in one leg and not the other irrespective of which ear is stimulated). The main finding of the Marsden et al study was abnormal interleg response asymmetry to galvanic vestibular stimulation in stroke patients only, the amplitude of the response being higher on the non-paretic side than on the paretic side, and higher on the non-paretic side than in controls. Since the changes in the reaction forces were observed early after vestibular stimulation (320-500 ms), the authors assumed that this initial response was likely to be purely vestibular in origin. Marsden et al also found that the degree of asymmetry induced by galvanic stimulation was correlated with the degree of corticospinal damage induced by transcranial magnetic stimulation. They concluded that MCA stroke may disrupt corticobulbar projection to brainstem output pathways which are involved in the vestibular control of balance. They suggested that stroke is associated with a lateralised deficit in the motor output stage of vestibular processing rather than in the sensory or spatial processing stages.

Postural rehabilitation must be guided by a better understanding of the postural disorders displayed by patients. There is no doubt that the paper by Marsden et al contributes to this knowledge. This new insight on the postural instability of standing stroke patients must now be confirmed and integrated with knowledge of the many other alterations and deficiencies which are involved in postural disorders caused by hemisphere strokes.

J Neurol Neurosurg Psychiatry 2005;76:621. doi: 10.1136/jnnp.2004.050468

Correspondence to: D Pérennou, Service de Rééducation Neurologique et INSERM ERIT M-207, Centre Hospitalo-Universitaire de Rééducation, 23 rue Gaffarel, 21034 Dijon cedex, France; dominic.perennou@chu-dijon.fr

Competing interests: none declared

\section{REFERENCES}

1 Pérennou DA, Bronstein AM. Balance disorders and vertigo after stroke: assessment and rehabilitation. In: Bogousslavsky J, Barnes $M$, Dobkin B, eds. Recovery after stroke. Cambridge: Cambridge University Press, 2005, pp 320-98.

2 Smith MT, Baer GD. Achievement of simple mobility milestones after stroke. Arch Phys Med Rehabil 1999;80:442-7.

3 Rode G, Tiliket C, Charlopain P, et al. Postural asymmetry reduction by vestibular caloric stimulation in left hemiparetic patients. Scand J Rehabil Med 1998;30:9-14.

4 Marsden JF, Playford ED, Day BL. The vestibular control of balance after stroke. J Neurol Neurosurg Psychiatry 2005;76:670-8.

5 Fitzpatrick RC, Day BL. Probing the human vestibular system with galvanic stimulation. J Appl Physiol 2004;96:2301-16. 


\section{Prognosis in the acute motor axonal form of Guillain-Barré syndrome}

\section{M Gabriel}

\section{Prognosis in axonal Guillain-Barré syndrome}

A primary axonal form of GuillainBarré syndrome was first described by Feasby and colleagues $^{1}$ in 1986. Initial indications were that this had a worse prognosis than demyelinating forms of the disease and it was suggested that recovery might require axonal regeneration along the entire length of the nerve fibre. In the intervening years it has become apparent that recovery from acute motor axonal neuropathy (AMAN) may actually be either rapid or slow. This is confirmed in a paper by Hiraga and colleagues (this issue, pp 719-22), ${ }^{2}$ and in addition they observed that in patients with slow recovery, clinical improvement to independent ambulation may continue for more than four years.

In the early 1990s, AMAN was characterised in northern China as a rapidly ascending tetraparesis with Wallerianlike degeneration of motor fibres at necropsy. ${ }^{3}$ This syndrome is most common in Japan and northern China, occurs uncommonly in Western countries, and is associated with Campylobacter jejuni (in 60-70\%), Haemophilus influenzae (in 10-20\%), and antiganglioside antibodies. Detailed ultrastructural study ${ }^{4}$ showed that the earliest changes were of myelin disruption at nodes of Ranvier, followed by macrophage extrusion into the periaxonal space at the paranode, with separation of the axon from the adjacent
Schwann cell membrane, and subsequent degeneration of both Schwann cell cytoplasm and axon.

It has become clear in more recent years that although AMAN progresses more rapidly to nadir, recovery times for AMAN and acute inflammatory demyelinating polyradiculoneuropathy (AIDP) are similar, and that in fact some patients with AMAN recover more quickly, ${ }^{5}$ especially if their illness is preceded by Haemophilus influenzae. Clinically, those who recover more rapidly are equally weak at the peak of their illness, though they are more likely to retain their tendon reflexes than those with AIDP.

Rapid recovery in AMAN in the current study was defined as an improvement of two or more GuillainBarré syndrome disability scale grades in the first four weeks e.g. from bedbound to independent ambulation, which occurred in $27 \%$ of the AMAN group. Such improvement would be incompatible with the primary pathology being Wallerian degeneration. Other explanations might be degeneration restricted to the distal motor nerve terminal where regeneration could occur quickly, immune mediated conduction block at the axonal membrane, or complement mediated damage to perisynaptic Schwann cells. This may imply additional pathological mechanisms distinct from those described ultrastructurally by Griffin and colleagues. The authors also identified a small number of patients who were unable to walk six months after the illness but all of whom continued to improve until they could walk independently in subsequent years.

It is possible that several different pathophysiological processes exist in AMAN to explain the dichotomous recovery patterns: reversible distal nerve failure at motor terminals or conduction block at nodes of Ranvier in association with rapid recovery, and Wallerian degeneration requiring axonal regrowth which may continue over many months and years. In AIDP, conventionally considered a demyelinating condition, severe lesions may result in axonal degeneration as well as myelin destruction, and a similar distinction dependent on disease severity may occur in AMAN. Certainly there is no unifying hypothesis to date, but it is encouraging that all forms seem to be potentially reversible.

J Neurol Neurosurg Psychiatry 2005;76:622. doi: 10.1136/jnnp.2004.055376

Correspondence to: Dr C M Gabriel, St Mary's Hospital, Praed Street, London W2 1NY, UK; carolyn.gabriel@st-marys.nhs.uk

Competing interests: none declared

\section{REFERENCES}

1 Feasby TE, Gilbert JJ, Brown WF, et al. An acute axonal form of Guillain-Barré polyneuropathy. Brain 1986; 109:1115-26.

2 Hiraga A, Mori M, Ogawara K, et al. Recovery patterns and long term prognosis for axonal Guillain-Barré syndrome. J Neurol Neurosurg Psychiatry 2005;76:719-22.

3 McKhann GM, Cornblath DR, Griffin JW, et al Acute motor axonal neuropathy: a frequent cause of acute flaccid paralysis in China. Ann Neurol, 1993 Apr, 33:333-42

4 Griffin JW, Li CY, Macko C, et al. Early nodal changes in the acute motor axonal neuropathy pattern of the Guillain-Barré syndrome. Neurocytol 1996;25:33-51.

5 Kuwabara S, Mori M, Ogawara K, et al Indicators of rapid clinical recovery in GuillainBarré syndrome. J Neurol Neurosurg Psychiatry 2001;70:560-2. 